\title{
HDV-Like Viruses
}

\author{
Jimena Pérez-Vargas ${ }^{1}{ }^{1}$, Rémi Pereira de Oliveira ${ }^{1}$, Stéphanie Jacquet ${ }^{2}$, Dominique Pontier ${ }^{2}$, \\ François-Loïc Cosset ${ }^{1, * \mathbb{D}}$ and Natalia Freitas ${ }^{1}$
}

check for

updates

Citation: Pérez-Vargas, J.; Pereira de Oliveira, R.; Jacquet, S.; Pontier, D.; Cosset, F.-L.; Freitas, N. HDV-Like

Viruses. Viruses 2021, 13, 1207.

https://doi.org/10.3390/v13071207

Academic Editor: Stefan Urban

Received: 5 May 2021

Accepted: 16 June 2021

Published: 23 June 2021

Publisher's Note: MDPI stays neutral with regard to jurisdictional claims in published maps and institutional affiliations.

Copyright: (C) 2021 by the authors Licensee MDPI, Basel, Switzerland. This article is an open access article distributed under the terms and conditions of the Creative Commons Attribution (CC BY) license (https:/ / creativecommons.org/licenses/by/ $4.0 /)$.
1 CIRI-Centre International de Recherche en Infectiologie, Université de Lyon, Université Claude Bernard Lyon 1, Inserm, U1111, CNRS, UMR5308, ENS Lyon, 46 allée d'Italie, F-69007 Lyon, France; jimena.perezvargas@gmail.com (J.P.-V.); remi.pereira_de_oliveira@ens-lyon.fr (R.P.d.O.); natalia.bezerra-de-freitas@ens-lyon.fr (N.F.)

2 LBBE UMR5558 CNRS-Centre National de la Recherche Scientifique, Université de Lyon 1-48 bd du 11 Novembre 1918, 69100 Villeurbanne, France; stephanie.jacquet@univ-lyon1.fr (S.J.);

Dominique.Pontier@univ-lyon1.fr (D.P.)

* Correspondence: flcosset@ens-lyon.fr

\begin{abstract}
Hepatitis delta virus (HDV) is a defective human virus that lacks the ability to produce its own envelope proteins and is thus dependent on the presence of a helper virus, which provides its surface proteins to produce infectious particles. Hepatitis B virus (HBV) was so far thought to be the only helper virus described to be associated with HDV. However, recent studies showed that divergent HDV-like viruses could be detected in fishes, birds, amphibians, and invertebrates, without evidence of any HBV-like agent supporting infection. Another recent study demonstrated that HDV can be transmitted and propagated in experimental infections ex vivo and in vivo by different enveloped viruses unrelated to $\mathrm{HBV}$, including hepatitis $\mathrm{C}$ virus (HCV) and flaviviruses such as Dengue and West Nile virus. All this new evidence, in addition to the identification of novel virus species within a large range of hosts in absence of HBV, suggests that deltaviruses may take advantage of a large spectrum of helper viruses and raises questions about HDV origins and evolution.
\end{abstract}

Keywords: HDV-like viruses; helper virus; origin; evolution

\section{Introduction}

Hepatitis D virus (HDV) was discovered 40 years ago in the liver of individuals chronically infected with hepatitis B virus (HBV) [1], a liver-specific pathogen present in ca. 250 million people. HDV is classified as the prototype and unique member of the Deltavirus genus. HDV forms enveloped particles with an average diameter of $36 \mathrm{~nm}$ that consists of cell-derived lipid vesicles harboring HBV surface proteins coating an inner ribonucleoprotein (RNP) [1]. This RNP is composed of a multimer of the HDV-encoded delta antigen (HDAg) [2] that is associated with one copy of a $1.7 \mathrm{~kb}$ long circular single strand HDV RNA exhibiting self-annealing properties [2]. Although HDAg was initially considered as a novel HBV antigen [3], it was later shown to be associated with a small RNA as a transmissible and defective agent that uses the HBV envelope glycoproteins (GP) for its propagation, hence reflecting its nature of obligate satellite of HBV [4]. For cell egress of its RNPs, HDV relies on the assistance of the helper HBV for the provision of GPs and a budding mechanism. Their envelopment subsequently allows targeting and entry of HDV particles to human hepatocytes via mechanisms that depend on the same host factors that govern the early entry events of HBV itself. Although HBV-related viruses (i.e., hepadnaviruses) are found in a number of mammals, HDV has thus far only been found in humans [1]. 


\section{Current Hypotheses of HDV Origin}

It is noteworthy that the origin of HDV is currently unknown. The HDV genome is unique and the smallest among animal viruses but shares some properties with some plant agents called viroids [5]. Indeed, the replication of HDV RNA involves the HDAg-mediated subversion of cellular RNA polymerase(s) (RNAP), such as Pol-II [6]. Both genomic HDV RNA and antigenomic RNA (its replication intermediate) strands include ribozyme autocatalytic, self-cleaving elements. However, most known viroids (i.e., pospiviroids) replicate by an asymmetrical rolling-circle mechanism. This pathway involves the generation of linear multimeric head-to-tail RNAs of negative polarity from circular RNAs of positive polarity by host RNAP(s). Interestingly, cells from highly divergent organisms express several HDV-like cellular ribozymes [7], which raises the possibility that HDV RNA originated from the cell transcriptome itself, in agreement with the finding that circular RNA (circRNA) species are abundant in cells [8]. The antigenomic RNA encodes for two forms of proteins based on RNA editing [9] and are referred to as small (S-HDAg, 195 amino acids) and large (L-HDAg, 214 amino acids) delta antigen [10]. S-HDAg promotes replication [11], whereas L-HDAg has been claimed to act as a dominant inhibitor of S-HDAg [12,13] and plays an essential role in virus assembly [14].

A cellular protein termed delta-interacting protein A (DIPA), initially identified as an HDAg ligand in a yeast two-hybrid screen [15], was proposed to represent a cellular ancestor of HDAg. DIPA and HDAg have shown $24 \%$ of protein sequence identity and $54 \%$ of similarity. Both proteins form oligomers through the coiled-coil domain, and it was suggested that the DIPA gene is a homolog of HDV and that capture of DIPA transcripts by a viroid-like sequence could have initiated the evolution of HDV [15].

Another hypothesis was that HDV RNA might have emerged as a consequence of an aberrant splicing event. A process known as mis-splicing, which produces RNA circles, was proposed; this occurs when a downstream $\left(5^{\prime}\right)$ splice donor is joined with an upstream $\left(3^{\prime}\right)$ splice acceptor site. However, an event of RNA-primed hairpin formation needs to occur in order to explain the base pairing of the HDV RNA $[16,17]$.

Thus, it is theoretically possible that HDV could have emerged in HBV-infected hepatocytes subsequent to the evolution of cellular circRNA forms becoming autonomously replicative [18] and for which the ribozyme and HDAg-coding RNA sequences may have arisen from the human transcriptome [15]. That HBV, a strictly liver-tropic human pathogen, only provides RNP envelopment and transmission functions to HDV would therefore explain why HDV has been exclusively detected in the liver of HBV-infected patients. However, HDV RNA can self-replicate in a much wider variety of cell types and species [19] and this raises the possibility that HDV may be transmitted through unorthodox means. Since HDV RNA can persist in the liver in the absence of HBV for several weeks [20], it is theoretically possible that its propagation could be triggered upon superinfection by other hepatitis viruses as well as by alternative infections with HBV-unrelated viruses [21]. A central component of these hypotheses is that HDV had been found, until recently, only in humans. Yet, several viruses closely related to HDV have now been detected in non-human species in the absence of any hepadnavirus [22-27]. Further studies are warranted to ascertain whether these agents represent satellite viruses dependent on a helper virus for their propagation, or alternatively, they are examples of a novel family of autonomous viroid-like agents in animals, whose transmission would occur by conventional receptormediated means.

\section{Discovery of HDV-Like Viruses}

For almost 40 years, HDV formed and was the sole member of the genus Deltavirus. Recently, however, virus-prospecting studies have discovered hepatitis D relatives (known as "deltaviruses") in a diverse range of animals (Table 1). Mining virus sequences from RNA libraries led to the discovery of two novel HDV-like sequences in ducks [22] and in Boa constrictors [23], indicating that deltaviruses likely exist in several taxa and that HDV has a far longer evolutionary history than previously anticipated. This hypothesis has 
been gaining ground, particularly after the identification of more deltavirus-like sequences in both vertebrates and invertebrates. Meta-transcriptomic browsing across a large set of RNA-Seq libraries from diverse animal hosts found four new HDV-like agents in a mixture of fishes (classes Actinopterygii, Chondrichthyes, and Agnatha), two amphibian species, and in termites [24]. These pioneering findings propelled more RNA-sequencing datasets searches for HDV-like sequences. Mammal deltaviruses have been identified in a neotropical rodent species Proechimys semispinosus (RDeV; [27]); in common vampire bats Desmodus rotundus (DrDeV-A and DrDeV-B); and in a liver transcriptome from a lesser dog-like bat Peropteryx macrotis (PmacDeV) [26]. Additional mammal deltavirus genomes were recovered from transcriptomes derived from white-tailed deer (Odocoileus virginianus; OvirDeV) and from captive-born Eastern woodchucks (Marmota monax; mmDeV) [25,26]. Three more deltavirus-like sequences have been also identified in birds, Taeniopygia guttata deltavirus (tgDeV), Serinus canaria-associated deltavirus ( $\mathrm{scDeV})$, and Erythrura gouldiae deltavirus (egDeV) [25].

In addition to the discovery of several novel deltavirus sequences in a broad host range spanning several taxonomic hosts, these metatranscriptomic approaches across different tissues and organs stressed important features of these novel deltaviruses infections; in particular, they highlighted tissue tropism differences among them and HDV. SDeV genome reads were found in NGS libraries prepared from the brain, blood, and liver [23]. Similarly, $\mathrm{RDeV}$, OvirDeV, and tgDeV infections do not seem to be limited to the liver, as the virus's sequence reads have been obtained from at least two different tissues. On occasion, data were consistent with a broad range or even systemic infection $[23,25,27]$.

Increased viral reads from the predicted transcribed regions, i.e., delta-like antigens coding region, have been used as a marker of active deltavirus replication/transcription [25], even though HDAg mRNA is the least prevalent viral RNA species during HDV infection [28]. Indeed, with some exceptions, there is a lack of experimental proof that the newly identified HDV-like sequences are capable of self-replicating. Furthermore, there are scarce data regarding their transmission potential within the animal population and limited knowledge about the molecular mechanisms of viral infection and propagation.

Using conventional RT-PCR, SDeV was found in the liver of four out seven individuals of the offspring of the SDeV positive parental founder snakes as well in a water python that lived in close proximity. Additionally, 3 out of 20 blood samples from snakes from a different breeder turned to be positive for $\mathrm{SDeV}$, suggesting that $\mathrm{SDeV}$ can be transmitted among animal populations [23]. However, further studies are required to ascertain whether $\mathrm{SDeV}$ is transmitted vertically or horizontally. In this respect, $\mathrm{RDeV}$ presence in the blood of adult animals only argues against vertical transmission [27]. Instead, it suggests that mammal deltaviruses similarly to HDV are passed horizontally and likely share similar routes of transmission.

Another unresolved question in the field is whether the newly reported deltaviruses can establish chronic infection like for the human HDV. Most likely the helper viruses involved and the efficacy of the host response combined shall determine the natural course of non-HDV deltaviruses infections. For tgDeV, $\mathrm{mmDeV}$, and $\mathrm{RDeV}$, it has been suggested that a host immune response can be mounted to suppress and clear deltavirus infections. For instance, the number $\mathrm{tgDeV}$ reads in birds correlated with the immunosuppressive effect of testosterone [25]. For one $\mathrm{mmDeV}$ positive woodchuck, the number of $\mathrm{mmDeV}$ genome reads over 27 weeks was compatible with acute infection [25], and the average viral load of RDeV was found to be decreased among animals with the highest antibody response against the rodent delta antigen (RDAg) [27].

Despite being highly divergent at the level of primary sequence, these non-human deltaviruses share many features with HDV. All characterized deltaviruses have a negativesense self-complementary circular RNA genome folded into the classical unbranched rod-like structure, including ribozymes [22,25-27]. While the presence of ribozymes has not been reported in the four metazoan HDV-like genomes identified by [24], a more recent study confirmed the presence of self-cleaving motifs in these HDV-like genomes [29]. HDV- 
like ribozymes have been recently mapped in the newt and fish HDV-like sequences [29]. Contrastingly, the toad and termite HDV-like genomes lacked analogous HDV-like ribozymes and instead showed the presence of conserved type III hammerhead ribozymes, which belong to an unrelated class of catalytic RNAs characteristic of plant genomes and plant subviral circular RNAs, such as some viral satellites and viroids [29].

Nevertheless, all of the newly identified sequences include a single open reading frame (ORF) representing the delta antigen encoding for proteins with sizes ranging from 180 to 225 amino acids (DAgs). It is noteworthy that a second ORF was identified in the genome of $\mathrm{SDeV}$ [23], though nothing is known about its function or if it is actually expressed during $\mathrm{SDeV}$ replication. Important functional domains and post-translational modification sites are conserved among all DAgs of the novel deltaviruses.

The most important and intriguing observation from these virus prospecting studies, based on epidemiological and serological evidence supported by metagenomic and transcriptomic findings, is the concept that deltaviruses infections and transmission seem to be independent of coinfection with an HBV-related hepadnavirus. With the exception of $\mathrm{mmDeV}$, all of the other HDV-like agents were found in the absence of hepadnaviruses [25]. In addition, the discovery of animal relatives of HDV, in particular in mammals, raises the hypothesis that hepatitis delta has jumped from a non-human animal to humans, and thus, it can be recognized as a zoonotic disease. Interestingly, all of the newly mammal deltaviruses were retrieved from hosts from the New World, the Americas, suggesting that HDV may have originated in the New World contrasting with the current hypothesis of HDV origin in Africa. Indisputably, the discovery of more mammalian deltaviruses and their hosts will help in better understanding HDV evolution, ascertaining their capacity for crossing species genetic barriers, and evaluating their potential to emerge in novel species and cause disease.

Table 1. Summary of Deltavirus infections.

\begin{tabular}{|c|c|c|c|c|c|c|}
\hline Deltavirus & Host & $\begin{array}{l}\text { CXXQ } \\
\text { Motif }\end{array}$ & $\begin{array}{c}\text { Putative } \\
\text { Co-Infecting } \\
\text { Viruses }\end{array}$ & $\begin{array}{c}\text { Tested Helper } \\
\text { Viruses } \\
\text { (In Vitro) }\end{array}$ & $\begin{array}{l}\text { HBsAg Usage } \\
\text { (In Vitro) }\end{array}$ & Refs \\
\hline $\mathrm{HDV}$ & Human & Yes & HBV & $\begin{array}{c}{ }^{\text {a }} \mathrm{HBV}^{\mathrm{b}} \mathrm{VSV},{ }^{\mathrm{c}} \mathrm{HCV}, \\
\text { d DENV, WNV, } \\
\text { e LCMV, }{ }^{\mathrm{f}} \mathrm{HMPV}\end{array}$ & Yes & {$[21,30-32]$} \\
\hline $\mathrm{aDeV}$ & ducks & no & Influenza A virus & nd & nd & [22] \\
\hline $\mathrm{RDeV}$ & $\begin{array}{l}\text { rodent } P \text {. } \\
\text { semispinosus }\end{array}$ & no & Hepacivirus & nd & nd & [27] \\
\hline $\mathrm{SDeV}$ & Boa constrictor & no & $\begin{array}{l}\text { Reptarenavirus, } \\
\text { hartmanivirus }\end{array}$ & $\begin{array}{c}\text { g UHV-2, UGV-1 } \\
\text { h HISV-1 } \\
\text { e LCMV, JUNV } \\
\text { f PUUV }\end{array}$ & no & {$[23,33]$} \\
\hline $\operatorname{tgDeV}$ & $\begin{array}{c}\text { Zebra finch } \\
\text { Taeniopygia guttata }\end{array}$ & no & none & nd & no & [25] \\
\hline $\mathrm{mmDeV}$ & $\begin{array}{l}\text { Eastern woodchuck } \\
\text { Marmota monax }\end{array}$ & no & $\begin{array}{l}\text { WHV, herpesvirus, } \\
\text { flavivirus, retrovirus }\end{array}$ & nd & no & {$[25,26]$} \\
\hline DrDeV & $\begin{array}{l}\text { Vampire bats } D . \\
\text { rotundus }\end{array}$ & no & $\begin{array}{l}\text { Herpesvirus, flavivirus, } \\
\text { retrovirus }\end{array}$ & nd & nd & [26] \\
\hline OvirDeV & $\begin{array}{l}\text { White-tailed deer } \\
\text { Odocoileus } \\
\text { virginianus }\end{array}$ & no & $\begin{array}{l}\text { Herpesvirus, flavivirus, } \\
\text { retrovirus }\end{array}$ & nd & nd & {$[25,26]$} \\
\hline
\end{tabular}


Table 1. Cont.

\begin{tabular}{ccccccc}
\hline Deltavirus & Host & $\begin{array}{c}\text { CXXQ } \\
\text { Motif }\end{array}$ & $\begin{array}{c}\text { Putative } \\
\text { Co-Infecting } \\
\text { Viruses }\end{array}$ & $\begin{array}{c}\text { Tested Helper } \\
\text { Viruses } \\
\text { (In Vitro) }\end{array}$ & $\begin{array}{c}\text { HBsAg Usage } \\
\text { (In Vitro) }\end{array}$ & $\begin{array}{c}\text { Refs } \\
\text { PmacDeV }\end{array}$ \\
\hline $\begin{array}{c}\text { Lesser dog-like bat } \\
\text { Peropteryx macrotis }\end{array}$ & no & $\begin{array}{c}\text { Herpesvirus, flavivirus, } \\
\text { retrovirus }\end{array}$ & nd & nd & {$[26]$} \\
\hline
\end{tabular}

List of newly identified deltaviruses and their hosts. Presence of the CXXQ motif in the virus antigen is indicated. The potential enveloped helper viruses based on either RNA-seq data, epidemiological or serological data are shown. Additionally, listed are the candidate helper viruses that were shown to successfully support deltaviruses assembly and infectivity in vitro. HBsAg usage, whenever known is indicated. a Hepadnavirues hepatitis B virus (HBV), woodchuck hepatitis virus (WHV), tent-making bat HBV (TBHBV) and Woolly monkey HBV (WMHBV); ${ }^{b}$ Vesiculovirus vesicular stomatitis virus (VSV); ${ }^{\mathrm{c}}$ Hepacivirus hepatitis C virus (HCV); ${ }^{\mathrm{d}}$ Flaviviruses

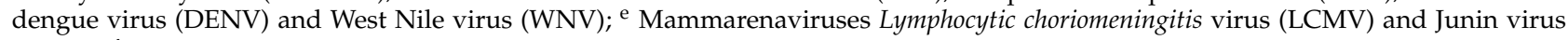
(JUNV); ${ }^{\mathrm{f}}$ Orthohantaviruses human metapneumovirus (HMPV) and Puumala virus (PUUV); ${ }^{\mathrm{g}}$ Reptarenaviruses UHV-2 and UGV-1; ${ }^{\mathrm{h}}$ Hartmanivirus HISV-1; nd-nondetermined.

\section{Phylogeny}

Based on the percentage of nucleotide identity of the genome, HDV was classified into eight genotypes, with two to four subtypes per genotype characterized by $>90 \%$ similarity over the entire genome sequence [34-36]. The distribution of various HDV genotypes was associated with geographical origins; however, the geographical distribution has changed over time, probably due to human migration patterns. Between genotypes, there can be as much as a $35 \%$ difference in nucleotide sequence [35].

The discovery of divergent HDV-like viruses from different hosts raises questions about the origin and evolution of HDV. Consequently, phylogenetic inferences and sequence analysis have been conducted each time a new deltavirus species was discovered. One of the first phylogenetic analyses estimated an average nucleotide identity of 29 to $46 \%$ and amino acid identity of 21 to $54 \%$ among all HDV genotypes and 7 of the newly reported deltaviruses [27]. RDeV was the single nonhuman mammalian deltavirus included in this study, and thus, not surprisingly, the phylogenetic reconstruction identified $\mathrm{RDeV}$ as the nonhuman deltavirus most closely related to human HDV clustering in the same clade with SDeV [27].

More recent phylogenetic analyses based on full-length protein sequences of DAg, including all HDV genotypes and the 12 HDV-like viruses described so far, revealed, however, different relationships among deltaviruses $[25,26]$. The mammalian deltaviruses DrDeVA, OvirDeV, and mmDeV share a common ancestor closely related to HDV (Figure 1), whereas $\mathrm{RDeV}$ is genetically related to PmacDeV clustering with $\mathrm{DrDeV}-\mathrm{B}$ and a clade consisting of $\mathrm{SDeV}, \mathrm{tgDeV}$, and $1 \mathrm{sDeV}$ [26]. Other related viruses (fish, amphibian and invertebrate HDV-like viruses) constitute basal lineages of the tree (Figure 1). Indeed, these latter viruses, which display the lowest degrees of amino acid sequence identity among deltaviruses [25], constitute divergent phylogenetic lineages being the most distant from other known deltaviruses. It is noteworthy that the basal nodes of the tree are not strongly supported, suggesting that other related deltaviruses (yet to be discovered) are likely missing. The current limited number of deltavirus species also prevents from drawing definitive conclusions of the origin of HDV. 


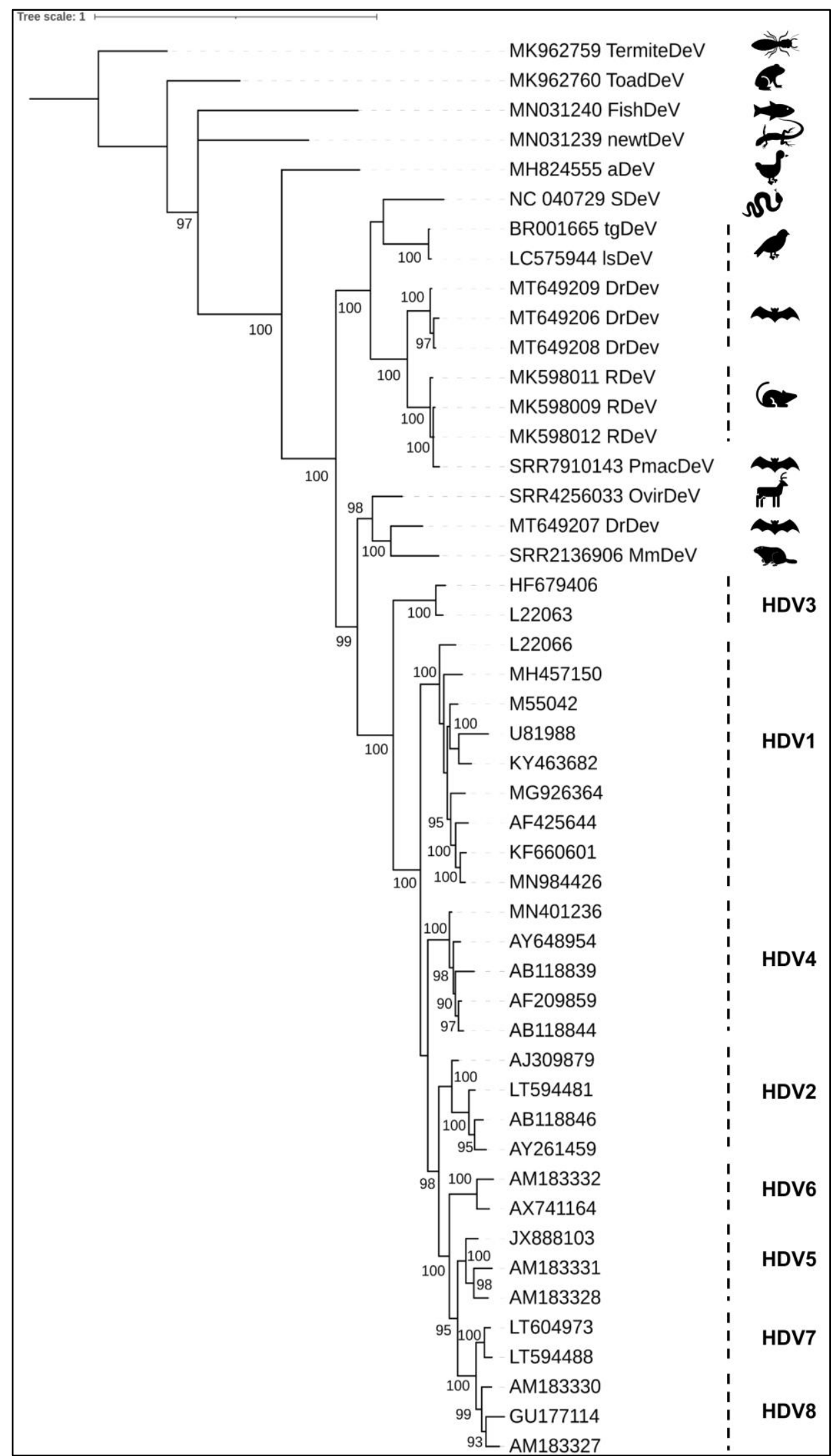

Figure 1. Bayesian phylogenetic tree of mammalian deltaviruses and HDV-like viruses. Small delta antigen (DAg) nucleotide sequences from published mammalian and invertebrate deltaviruses, including representative HDV clades, were aligned using the translated protein option (without the 
trust insertion -F) implemented in PRANK $[37,38]$. Phylogenetic reconstruction of DAg was carried out using the resulting protein alignment and the best amino acid substitution model $(\mathrm{LG}+\mathrm{F}+\mathrm{G})$ inferred by Smart Model Selection [39]. The Bayesian analysis was carried out with MrBayes, using 2,000,000 generations with a tree sampling every 1000 generations and a burn-in of 500 trees. The final tree was rooted with the termite HDV-like virus. Tree edition was performed with iTOL [40].

With the discovery of new HDV-like viruses, new phylogenetic analyzes will allow a better understanding and reconstruction of the evolutionary history of animal deltaviruses and their relationships with their respective hosts.

\section{Virus Replication, Genome Editing, and Viral Proteins Expression}

HDV virion encloses a circular, single-stranded negative-sense RNA molecule associated with an internal core delta antigen surrounded by envelope glycoproteins. The full-length circular genome for HDV is approximately 1680 nucleotides, whereas for HDVlike agents, it ranges between 1592 and 1735 nucleotides. The GC contents of these novel HDV-like agents range between 46 and 58 percent, which is slightly lower than the human HDV with a GC content of around 60 percent [22-26]. The circular nature and self-annealing properties of HDV RNAs are reminiscent of some plant viroids and are important for both genome replication and RNP assembly upon interaction with HDAg proteins [41-43]. Most RNA viruses encode their own replicases or RNA-dependent RNA polymerases (RdRp) essential for viral genome replication. However, HDV does not carry an RdRp gene to replicate its genome. It rather makes use of cellular DNA-dependent RNA polymerases to replicate its genome. Since HDV genomic RNA has a negative or anti-messenger polarity, during replication, three different forms of RNA are made: linear $0.8 \mathrm{~Kb}$ messenger RNA (mRNA) of antigenomic polarity harboring a $5^{\prime}$-cap and a $3^{\prime}$-polyadenylated tail $[28,44]$, circular genomic RNA, and circular complementary antigenomic RNA, which are synthetized via a rolling-circle mechanism similar to that proposed for some viroids $[45,46]$. The rolling-circle mechanism involves unidirectional replication of nucleic acids to form multiple copies of the circular genome using cellular RNA polymerases. The process is not completely understood, but only the genomic RNA is assembled into HDV particles, even if both genomic and antigenomic RNA replication is detected in the nucleus, suggesting that there is a selective export of genomic RNA to the cytoplasm [47]. Nevertheless, in vitro antigenomic RNA can be secreted as long as the large hepatitis delta antigen, L-HDAg, and HBsAg are coexpressed [48].

A ribozyme, a self-cleaving RNA sequence, resides in the viroid-like sequence of the HDV genome that cleaves a linear form of multiple-copy length of the viral genome or antigenome into monomeric units that are then circularized to complete the replication cycle. All described HDV-like agents contain self-cleaving motifs from either the HDV or the hammerhead class $[22,23,26,27,29]$. In vitro transcription and self-cleavage analysis confirmed the cleaving activity for these motifs in the newt, fish, toad, and termite HDV-like RNAs [29]. The cleaving activity for the other identified ribozymes has not yet been tested.

Many cell culture model systems have been developed to study HDV genome replication and regulation of viral gene expression. One of the most simplified systems relies on the transfection of cultured cell lines with larger than unit-length cDNA clones (usually dimers or trimers) of HDV sequences [11]. HDV cDNA vectors have been constructed to produce either genomic or antigenomic RNA and both kinds of recombinants have been shown to be competent to initiate genome replication [11,49]. In these transfection assays, the first round of HDV RNA synthesis is DNA-directed under the control of a heterologous promoter, followed by subsequent rounds of RNA-directed RNA replication that is absolutely dependent on S-HDAg [6]. RNA-directed RNA replication can be easily assayed by Northern analysis with a probe specific for the product of replication, i.e., viral RNA of opposite polarity to the RNA transcribed from the cDNA template, and viral gene expression by Western blot and immunofluorescence [11,49]. 
Using an analog approach, it was shown that RDeV could initiate replication in Huh7 cells transfected with a construct carrying a head-to-tail dimer of the full RDeV genome in genomic orientation as demonstrated by the presence of the marker of on-going RNAdirected RNA replication, i.e., viral RNA of antigenomic polarity and S-RDAg as early as 4 days post-transfection [27].

HDV uses RNA editing as a mechanism to switch from genome replication to packaging. During replication, a fraction of the antigenome RNA is edited by ADAR1 such that the adenosine within the amber stop codon that terminates the S-HDAg ORF is deaminated to inosine (Figure 2). The stop codon is thus changed to tryptophan and the ORF is extended by additional 19 or 20 amino acids to yield L-HDAg. Within the 19 C-terminal amino acids unique to L-HDAg, a nuclear export signal (NES) has been identified [50]. It also contains a conserved CXXQ box motif that is post-translationally modified with an isoprenyl anchor [51]. These lipid moieties, a farnesyl in the case of L-HDAg, are typically involved in mediating protein-membrane and protein-protein interactions. Indeed, farnesylation of L-HDAg enables the interaction between HDV RNPs and tryptophan residues of the small cytosolic loop of HBsAgs [52] and thus is required for virion assembly. It is also implicated in the mechanism by which L-HDAg inhibits HDV RNA replication [53]. Interestingly, farnesyl-mediated targeting to cellular membranes of L-HDAg seems to assist HDV assembly with envelope proteins from unorthodox helper viruses [21].

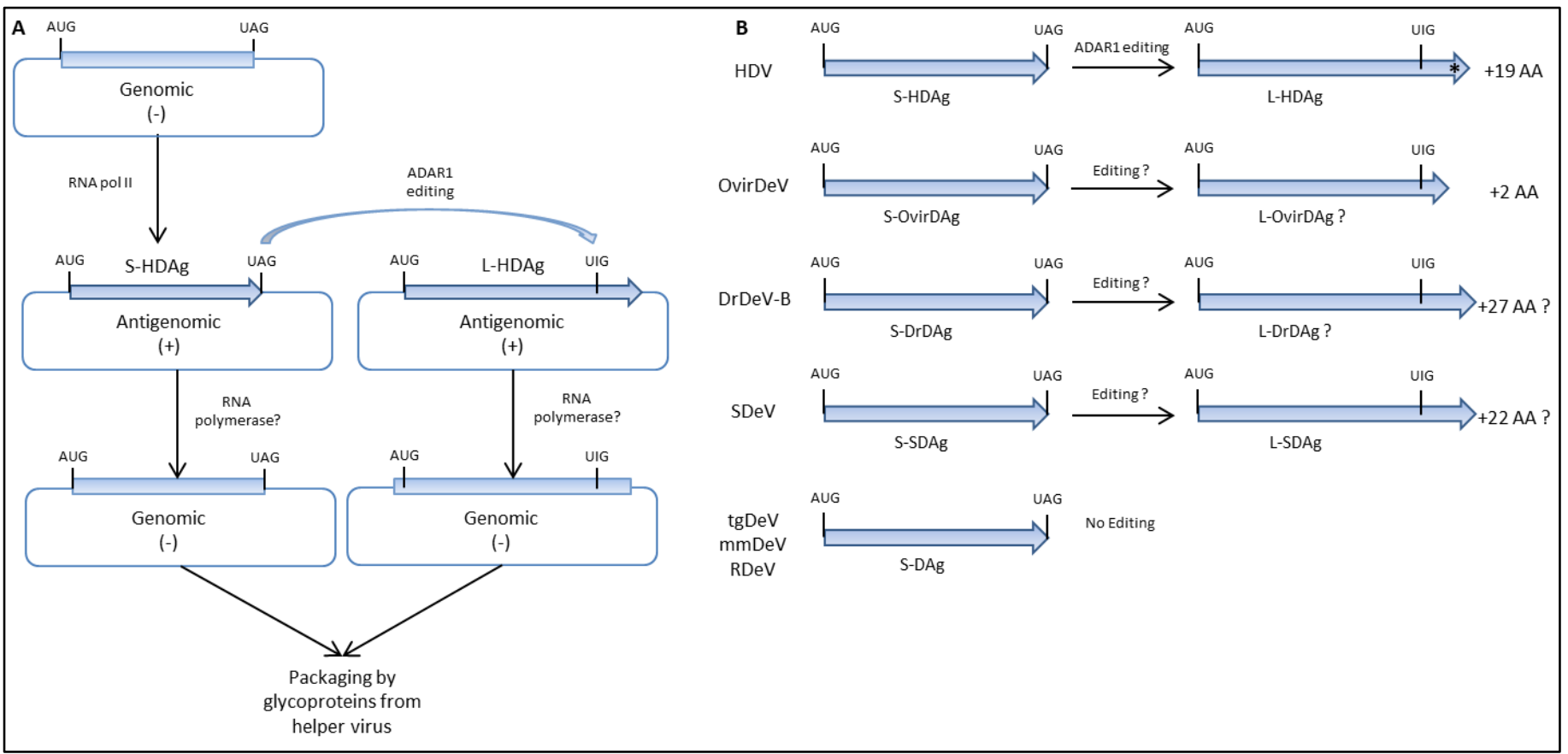

Figure 2. Schematic representation of RNA editing during deltaviruses replication (A) and putative L-DAgs generated via ADAR1 (B). The incoming viral genome, a circular ssRNA molecule of negative polarity (genomic RNA), serves as a template for the synthesis of DAg encoding mRNAs and circular RNAs of antigenomic polarity. The mRNA produced at the early stages of deltaviruses replication contains an amber stop codon (UAG) and produces the small form of DAgs. In a fraction of the antigenomic RNAs, the adenosine in the amber stop codon is deaminated to inosine (UIG) by the host RNA editing enzyme ADAR1. The edited mRNAs contain a tryptophan (W) codon instead of the amber stop codon and additional codons are translated to yield the large form of DAgs. The asterisk depicts the presence of a CXXQ motif in the C-terminus of L-HDAg required for isoprenylation.

Similar to S-HDAg, the RDAg ORF also terminates with a UAG amber stop codon [27]. A similar A-to-I editing of the RDeV antigenome RNA could eliminate the stop codon of the S-RDAg ORF and extend the ORF by additional 19 amino acids. In Huh7 cells transfected with HDV cDNAs, L-HDAg levels are barely detectable during the first post-transfection days but then progressively increase relative to S-HDAg [43]. Western blot analysis showed 
no signs of a large RDAg antigen (L-RDAg) expression in Huh7 cells transfected with a dimeric RDeV cDNA construct monitored every 2 days for a total period of 10 days [27]. Consistently, there was also no evidence of RNA editing using RNA-seq data of the blood and organ samples of infected animals and RNA sequences extracted from the transfected cells. Overall, it seems that during RDeV infection, antigenomic editing and L-RDAg production do not occur [27].

In contrast to RDeV, two species of NGS reads were detected for OvirDeV at the second position of the stop of the S-OvirDAg (UAG). The base-editing frequency measured was $0.4 \%$ with all edited sequences showing a $G$ instead of the consensus $A$. While this A-to-I nucleotide variation may suggest RNA editing by ADAR1, the generated ORF would be extended by two amino acids only due to the presence of a stop codon immediately after [25]. However, there are no experimental data addressing OvirDeV editing or large antigen expression.

A putative 28-amino acid carboxyl-terminal extension was also identified in the bat deltavirus (DrDeV) genome [26], although there is no experimental evidence of $\mathrm{DrDeV}$ RNA editing or large antigen expression. Even though RNA editing could occur, the putative resultant protein, as well as all other deltaviruses putative large antigens lack a farnesylation motif. For $\mathrm{mmDeV}$ and $\mathrm{tgDeV}$, no nucleotide variations could be identified among the stop codons of the viral genes, suggesting that these two viruses do not undergo RNA editing during replication [25]. A single form of the virus antigen was detected after transfection with $\mathrm{mmDeV}$ and $\mathrm{tgDeV}$ replication competent cDNA constructs [25].

Sequence analysis of the $\mathrm{SDeV}$ genome revealed a potential editing site at the UAG amber stop codon of the S-SDAg. RNA editing would increase the ORF by 22 additional amino acids, and thus, two bands with estimated molecular weights of $22.7 \mathrm{kDa}$ and $25.6 \mathrm{kDa}$ should be detected by Western blotting in the homogenates of tissue samples from infected snakes. Indeed, an anti-S-SDAg antiserum detected two bands with molecular weights that are approximate to the theoretical estimates for S- and L-SDAg in liver samples of infected snakes but mainly only the large form was detected in the brain samples [23]. Whether the two bands observed are tissue dependent and result from RNA editing still remains unclear because the study does not provide NGS data in support of antigenomic virus RNA editing. Additional studies are thus awaited to address the differences in SDAg processing and maturation. Another peculiarity concerning SDAg is its prominent cytoplasmic staining despite the presence of putative nuclear localization signals (NLSs) in its amino acid sequence in different tissues of infected Boa constrictor snakes [23]. SDAg distribution pattern was further analyzed by transfection of boid and monkey kidney cell lines, I/1Ki and Vero E6 cells, respectively. In both cell lines, SDAg was predominantly found in the cytoplasm independently of the orientation of the SDeV cDNA used to initiate virus replication [33]. Similar results were obtained in human kidney and cervical HeLa cells and in snake lung cells. However, a nuclear staining resembling HDAg was also found in human lung cells as well as in snake and human heart and liver cell lines.

In contrast to HDV, RNA editing seems not to be the mechanism regulating the switch from replication to viral RNPs packaging for the newly identified deltaviruses (Figure 2). Additionally, the lack of large antigen expression and farnesylation should prevent the interaction with hepadnaviral envelope proteins.

HDV RNA and HDAg proteins interact to form the HDV ribonucleoprotein (RNP), the delta antigens form multimers, and the HDV RNA wraps around such multimers. It has been suggested that the multimers may be predominantly octamers of the delta antigen and that the genomic RNA, which is partially double-stranded, wraps around the octamers in a way analogous to how double-stranded host DNA wraps around histone octamers $[2,54]$. The predicted coiled-coil domains of HDAgs that facilitate multimerization and replication, found at the $\mathrm{N}$-terminal region between amino acids 22 and 44 , were also found in HDV-like agents [22,27]. 


\section{Helper Virus's Functions}

The envelope proteins of HBV are the only contribution of the helper virus to the HDV life cycle. The determinants governing HBV tissue and species tropism also dictate HDV tropism to human hepatocytes. HBV and HDV entry requires the specific interaction with the preS1 domain of viral L-HBsAg. Despite sharing the same envelope and using the same host receptor, HBV and HDV show different requirements of host factors to establish productive infections. In contrast to $\mathrm{HBV}, \mathrm{HDV}$ infection is predominantly determined at the receptor level [55]. Ectopic expression of human NTCP is enough to confer cells from different tissue and species susceptibility for HDV, whereas additional host factors besides NTCP are required for successful HBV infection [55,56]. HDV RNA replication is entirely independent of the helper virus. Indeed, HDV RNA can persist in the liver of humanized mice in the absence of HBV for several weeks and potentially be rescued by a later HBV infection [20]. Additionally, primary Sjögren's syndrome patients were reported to present HDV antigen and RNA in salivary glands in the absence of HBsAg or HBV antibodies [57].

For budding HDV takes advantage of the quite inefficient HBV assembly process that produces relatively huge amounts of empty particles [58]. The budding process involves interaction between the farnesylated Cys-211 of L-HDAg with the cytosolic loop of the HBsAg, and it can be independent of active HBV replication within the same cell. In line with this view, infectious HDV particles can be produced in cells lacking markers of ongoing HBV replication but expressing HBV envelope proteins from integrated virus DNA into the host cell genome [59]. Experimentally, HDV can also make use of envelope proteins of WHV to produce infectious progeny. These particles were shown to be capable of infecting both primary human hepatocytes $(\mathrm{PHH})$ and woodchuck primary hepatocytes (WPH), whereas HDV assembled with HBV cannot enter WPH [32]. Recently, we demonstrated that HDV can be transmitted and propagated in experimental ex vivo and in vivo infections by different enveloped viruses unrelated to $\mathrm{HBV}$, including hepatitis $\mathrm{C}$ virus (HCV), flaviviruses such as Dengue and West Nile viruses, and Vesiculovirus [21].

Similar to HDV, the newly identified deltaviruses RNA can self-replicate in a wide variety of cell types and species $[19,23,27,33]$, indicating that while deltavirus replication is not limited to any particular cell type, it is restricted by the envelope glycoproteins borrowed from the helper virus. As for HDV, mmDeV and SDeV cDNA transfection can lead to a persistent infection that survives cell division without the requirement of a helper virus $[25,33]$. While there is no information available describing the competence of snake cell lines supporting HDV replication, SDeV persistence lasted up to 6 months post-transfection, at least two times longer than HDV in Huh7 cells [60]. This persistence seems to be cell type and/or species dependent since SDeV could not establish persistent infection in human cells, suggesting the existence of virus-intrinsic and/or host-specific determinants determining deltavirus replication. Even though direct cell-cell SDeV spread cannot be excluded without control experiments, it seems that $\mathrm{SDeV}$ persistence results from clonal expansion, rather than cell-free virus transmission as the supernatants from the chronically infected snake cells were incapable of passing SDeV infection to naïve cells [33].

While the potential candidate helper viruses for these novel deltaviruses are currently unknown, the lack of a farnesylation motif (CXXQ), required for HDV assembly and release in the C-terminal region of the novel deltaviruses antigens (Table 1), highlights new and alternative mechanisms for deltaviruses envelopment and cell egress [25,27].

\section{Candidate Helper Viruses}

HDV transmission among the human population requires HBV envelope proteins. Likely, the transmission of the newly reported deltaviruses in animal populations might also be dependent on coinfecting helper viruses to provide envelope proteins to copackage deltaviruses RNPs and assist dissemination.

The final stage of the HDV life cycle, that is, the incorporation of the newly replicated RNA genome associated with delta antigens into enveloped virions, can be easily accom- 
plished by coinfection with a helper virus or by cotransfection with constructs encoding the envelope proteins of hepadnavirus belonging to the genus Orthohepadnavirus.

For decades, only two hepadnavirus genera were known: genus Orthohepadnavirus, which infects mammalian species, and genus Avihepadnavirus, which infects avian species. Since 2019, however, five hepadnavirus genera are recognized [61]. The three newly defined genera include the herpetohepadnaviruses (genus Herpetohepadnavirus), which infect amphibians and reptiles, as well as two highly distinct groups that infect fishes-the metahepadnaviruses (genus Metahepadnavirus) and the parahepadnaviruses (genus Parahepadnavirus) [62-64].

Since HDV has to coexist with HBV for propagation, a first assumption would be that HDV should have evolved in animals that were susceptible to infection by hepadnaviruses, namely, two of the newly discovered deltaviruses, $\mathrm{aDeV}$ and $\mathrm{mmDeV}$, were retrieved from RNA-seq libraries of ducks and eastern woodchucks that are natural hosts for hepadnaviruses $[22,25]$ and can be coinfected with their respective Orthohepadnavirus species, the duck hepatitis B virus (DHBV) and the woodchuck hepatitis virus (WHV).

$\mathrm{SDeV}$ isolated from brain homogenates was shown to be able to establish persistent infection in boid kidney cells (I/1Ki cells). The same metatranscriptomic analyses that led to SDeV discovery revealed that the infected snakes had no traces of coinfection with HBV-like viruses. Instead, NGS analyses retrieved sequences of the L and S RNA segments of reptarena- and hartmanviruses [33], suggesting that arenaviruses could act as $\mathrm{SDeV}$ helper viruses and provide the envelope glycoproteins required for the formation of infectious particles. Indeed, staining of SDeV infected cells with antibodies against SDAg and anti-, reptarena-, or hartmanviruses nucleoprotein (NP) suggested the SDeV-brain homogenate to be coinfected with all three viruses. In vitro superinfection of permanently SDev-infected snake cells with reptarenaviruses (UHV-2 and UGV-1), and the hartmanvirus HISV-1 confirmed that SDeV could use arenaviruses as helper viruses to produce $\mathrm{SDeV}$ infectious particles capable of infecting naïve cells. Furthermore, it was demonstrated that transfection of plasmids encoding the glycoproteins of HISV-1, UGV-1, the othohantavirus Puumala virus (PUUV), the lymphocytic choriomeningitis virus (LCMV), and Junin virus (JUNV) was enough to drive the production of $\mathrm{SDeV}$ infectious particles but not a synthetic gene covering the $\mathrm{L}, \mathrm{M}$ and $\mathrm{S}$ surface proteins of HBV. However, the infectivity of SDeV particles pseudotyped with HBV glycoproteins was assessed on snake kidney cells, an unsuitable cell culture model to evaluate HBV-envelope-mediated entry, which has limited cellular and host tropism [33].

Many in vitro and in vivo infection studies demonstrated that woodchuck hepatitis virus (WHV), a hepadnavirus related to HBV, could provide helper activity for HDV [32,65]. As one of the newly identified deltaviruses, $\mathrm{mmDeV}$ was identified in the liver transcriptome from Eastern woodchucks (Marmota monax) experimentally infected with WHV and in two sequence read archive (SRA) data derived from PMBCs of animals negative for antibodies against WHV as well as WHV DNA [25]. The liver tropism and coinfection with $\mathrm{mmDeV}$ and WHV could suggest a well-established satellite/helper relationship between another mammalian deltavirus and a hepadnavirus. However, WHV was experimentally inoculated in these animals, and therefore, the presence of both viruses in the liver may not reflect important virus association for $\mathrm{mmDeV}$ transmission. Additionally, $\mathrm{mmDeV}$ does not seem to utilize the A-to-I RNA editing mechanism to extend the viral ORF and neither carries a CXXQ signal required for farnesylation [25]. In line with the idea that hepadnaviruses do not provide helper activity for $\mathrm{mmDeV}$ transmission, it was demonstrated that HBsAgs were not capable of supporting $\mathrm{mmDeV}$ or $\mathrm{tgDeV}$ assembly and infectivity [25].

Despite the lack of experimental evidence, the libraries dominated with WHV also contained reads matching viral sequences of Herpesvirus, Flavivirus, Retrovirus, and Poxvirus, representing potential sources of helper activity. Interestingly, Poxvirus hits were identified in libraries where four other deltaviruses have been identified, i.e., DrDeV-A, 
DrDeV-B, PmacDeV, and OvirDeV, which was hence considered as a potential ecological association worth to be evaluated in future studies [26].

As hepacivirus infections are highly prevalent in rodents and since $\mathrm{HCV}$, in particular, can provide envelope proteins for HDV and support its transmission in vivo [17,21], the question of whether hepacivirus could support $\mathrm{RDeV}$ infection has been raised. Although 26 out of the $30 \mathrm{RDeV}$ RNA-positive rodents were also positive for hepacivirus, statistical analyses did not recognize any significant association between $\mathrm{RDeV}$ and hepacivirus infections in the 763 animals sampled [27]. Whereas the suggested lack of dependency between $\mathrm{RDeV}$ and hepacivirus infections can be explained by the absence of a farnesylation motif in the rodent delta antigen, the possibility of alternative enveloping mechanisms, enabling the usage of hepacivirus envelope proteins by $\mathrm{RDeV}$, remains to be investigated. A noteworthy finding is that both $\mathrm{HDV}$ and $\mathrm{SDeV}$ can be assembled with Hantaviruses and Arenaviruses envelope proteins strengthens this hypothesis [21,33]. It suggests that even HDV RNPs incorporation into enveloped particles can be independent of host-mediated prenylation of L-HDAg and also implies that divergent deltaviruses could share alternativeenveloping mechanisms. Future research studies will certainly address these and other questions. For instance, do retroviruses support non-human deltaviruses assembly and infectivity contrasting with their inability to sustain HDV propagation [21]?

\section{Conclusions}

Undoubtedly, the discovery of HDV-like viruses in distantly related species other than humans and their detection in the absence of HBV or hepadnavirus infection, as well as the evidence that HDV and HDV-like virus can indeed use envelope glycoproteins from viruses of diverse genera for transmission, open numerous questions over the origin of deltaviruses and the nature of the relationship between HDV and HBV. This suggests that HDV-like viruses have been associated with animals long before their first appearance in humans [36] and during their evolutionary history. It is still possible that the ancestor of deltaviruses emerged from cellular RNAs; however, it seems likely that it would have happened much earlier in evolution than previously thought. However, we cannot discard the hypothesis that a common ancestor of deltaviruses could be found among viroids of plants, as they show the highest similarities with deltaviruses $[66,67]$. Since a deltavirus has already been identified in termites [24], one possibility is that the deltavirus ancestor was transmitted to animals from plants, since the presence of viroids in aphids feeding on infected plants was already shown [68], which could support the above hypothesis. The phylogeny suggests that the mammalian deltaviruses are closer to HDV common ancestor, whereas avian, reptile, fish, or amphibian HDV-like viruses are more distant. Nevertheless, the taxonomic distance among the deltavirus hosts does not fully support the hypothesis of virus-host codivergence. Based on the phylogenetic tree, it rather supports cross-species transmissions in mammals. The discovery of HDV-like viruses in other species is necessary to have more elements to answer the questions regarding the evolution of deltaviruses. It is also tempting to speculate that deltaviruses had an origin in other species different than humans and use other helper viruses than HBV-related viruses; yet, in humans, HDV may have found HBV as its best partner.

It is noteworthy that three clinical studies have now been performed to verify the possibility of HDV infection in patients only coinfected with HCV [69-71]. Although one study from our group provided preliminary evidence of HDV exposure in chronically HCV-infected patients in the absence of ongoing or past HBV infection [70], overall, these studies also highlighted the rarity of HDV and HCV coinfection events at least in such patients.

Altogether, these studies pave the way to a better understanding of the origin of HDV and enlarge the deltaviruses research field. They also raise several questions concerning the immunological and virologic relations between HDV and HBV or alternative helper viruses, and the exact pathogenesis and tissue tropism of HDV. Perhaps one of the most important questions is if HDV can be transmitted in humans by other helper viruses than 
HBV. Additionally, if HDV has a wider range of potential coinfections than previously thought, what could be the clinical relevance and pathobiological implications?

Author Contributions: Conceptualization, J.P.-V., F.-L.C., N.F.; writing—original draft preparation, J.P.-V., F.-L.C., N.F.; writing-review and editing, J.P.-V., R.P.d.O., S.J., D.P., F.-L.C., N.F.; visualization, N.F.; supervision, F.-L.C. and N.F.; project administration, F.-L.C.; funding acquisition, D.P., F.-L.C. and N.F. All authors have read and agreed to the published version of the manuscript.

Funding: The laboratory of F.-L.C. is supported by the Agence Nationale de la Recherche sur le SIDA et les Hépatites Virales I Maladie Infectieuses Emergentes (ANRS I MIE), the IDEXLYON (ANR-16-IDEX-0005) and the LabEx Ecofect (ANR-11-LABX-0048) of the "Universite de Lyon" (http:/ / ecofect.universite-lyon.fr/, accessed on 25 April 2021), within the program "Investissements d'Avenir" (ANR-11-IDEX-0007) operated by the ANR.

Institutional Review Board Statement: Not applicable.

Informed Consent Statement: Not applicable.

Conflicts of Interest: The authors declare no conflict of interest.

\section{References}

1. Rizzetto, M.; Hoyer, B.; Canese, M.G.; Shih, J.W.; Purcell, R.H.; Gerin, J.L. Delta Agent: Association of delta antigen with hepatitis B surface antigen and RNA in serum of delta-infected chimpanzees. Proc. Natl. Acad. Sci. USA 1980, 77, 6124-6128. [CrossRef] [PubMed]

2. Lin, B.C.; Defenbaugh, D.A.; Casey, J.L. Multimerization of Hepatitis Delta Antigen Is a Critical Determinant of RNA Binding Specificity. J. Virol. 2010, 84, 1406-1413. [CrossRef] [PubMed]

3. Rizzetto, M.; Canese, M.G.; Arico, S.; Crivelli, O.; Trepo, C.; Bonino, F.; Verme, G. Immunofluorescence Detection of New Antigen-Antibody System (Delta/Anti-Delta) Associated to Hepatitis-B Virus in Liver and in Serum of Hbsag Carriers. Gut 1977, 18,997-1003. [CrossRef]

4. $\quad$ Rizzetto, M.; Canese, M.G.; Gerin, J.L.; London, W.T.; Sly, D.L.; Purcell, R.H. Transmission of the Hepatitis B Virus-Associated Delta Antigen to Chimpanzees. J. Infect. Dis. 1980, 141, 590-602. [CrossRef] [PubMed]

5. Wang, K.S.; Choo, Q.L.; Weiner, A.J.; Ou, J.H.; Najarian, R.C.; Thayer, R.M.; Mullenbach, G.T.; Denniston, K.J.; Gerin, J.L.; Houghton, M. Structure, Sequence and Expression of the Hepatitis Delta (Delta) Viral Genome. Nature 1986, 323, 508-514. [CrossRef]

6. Lai, M.M.C. RNA Replication without RNA-Dependent RNA Polymerase: Surprises from Hepatitis Delta Virus. J. Virol. 2005, 79, 7951-7958. [CrossRef]

7. Riccitelli, N.; Lupták, A. HDV Family of Self-Cleaving Ribozymes. Prog. Mol. Biol. Transl. Sci. 2013, 120, 123-171. [CrossRef]

8. Memczak, S.; Jens, M.; Elefsinioti, A.; Torti, F.; Krueger, J.; Rybak-Wolf, A.; Maier, L.; Mackowiak, S.; Gregersen, L.H.; Munschauer, M.; et al. Circular RNAs are a large class of animal RNAs with regulatory potency. Nature 2013, 495, 333-338. [CrossRef]

9. Casey, J.L.; Gerin, J.L. Hepatitis-D Virus-RNA Editing-Specific Modification of Adenosine in the Anti-genomic RNA. J. Virol. 1995, 69, 7593-7600. [CrossRef]

10. Weiner, A.J.; Choo, Q.L.; Wang, K.S.; Govindarajan, S.; Redeker, A.G.; Gerin, J.L.; Houghton, M. A Single Antigenomic Open Reading Frame of the Hepatitis Delta Virus Encodes the Epitope(S) of Both Hepatitis Delta Antigen Polypeptides P24-Delta and P27-Delta. J. Virol. 1988, 62, 594-599. [CrossRef]

11. Kuo, M.Y.P.; Chao, M.; Taylor, J. Initiation of Replication of the Human Hepatitis-Delta Virus Genome from Cloned DNA-Role of Delta-Antigen. J. Virol. 1989, 63, 1945-1950. [CrossRef]

12. Modahl, L.E.; Lai, M.M.C. The Large Delta Antigen of Hepatitis Delta Virus Potently Inhibits Genomic but Not Antigenomic RNA Synthesis: A Mechanism Enabling Initiation of Viral Replication. J. Virol. 2000, 74, 7375-7380. [CrossRef] [PubMed]

13. Chao, M.; Hsieh, S.Y.; Taylor, J. Role of 2 Forms of Hepatitis Delta Virus-Antigen-Evidence for a Mechanism of Self-Limiting Genome Replication. J. Virol. 1990, 64, 5066-5069. [CrossRef] [PubMed]

14. Chang, F.L.; Chen, P.J.; Tu, S.J.; Wang, C.J.; Chen, D.-S. The large form of hepatitis delta antigen is crucial for assembly of hepatitis delta virus. Proc. Natl. Acad. Sci. USA 1991, 88, 8490-8494. [CrossRef] [PubMed]

15. Brazas, R.; Ganem, D. A Cellular Homolog of Hepatitis Delta Antigen: Implications for Viral Replication and Evolution. Science 1996, 274, 90-94. [CrossRef]

16. Diener, T. The viroid: Biological oddity or evolutionary fossil? Adv. Virus Res. 2001, 57, 137-184. [CrossRef]

17. Dinter-Gottlieb, G. The Unique Hepatitis Delta Virus; Springer: Berlin/Heidelberg, Germany, 1995.

18. Taylor, J.M. Host RNA circles and the origin of hepatitis delta virus. World J. Gastroenterol. 2014, 20, 2971-2978. [CrossRef]

19. Polo, J.M.; Jeng, K.S.; Lim, B.; Govindarajan, S.; Hofman, F.; Sangiorgi, F.; Lai, M.M.C. Transgenic Mice Support Replication of Hepatitis-Delta Virus-RNA in Multiple Tissues, Particularly in Skeletal-Muscle. J. Virol. 1995, 69, 4880-4887. [CrossRef] 
20. Giersch, K.; Helbig, M.; Volz, T.; Allweiss, L.; Mancke, L.V.; Lohse, A.W.; Polywka, S.; Pollok, J.M.; Petersen, J.; Taylor, J.; et al. Persistent hepatitis D virus mono-infection in humanized mice is efficiently converted by hepatitis B virus to a productive co-infection. J. Hepatol. 2014, 60, 538-544. [CrossRef]

21. Perez-Vargas, J.; Amirache, F.; Boson, B.; Mialon, C.; Freitas, N.; Sureau, C.; Fusil, F.; Cosset, F.-L. Enveloped viruses distinct from HBV induce dissemination of hepatitis D virus in vivo. Nat. Commun. 2019, 10, 2098. [CrossRef]

22. Wille, M.; Netter, H.J.; Littlejohn, M.; Yuen, L.; Shi, M.; Eden, J.-S.; Klaassen, M.; Holmes, E.C.; Hurt, A.C. A Divergent Hepatitis D-Like Agent in Birds. Viruses 2018, 10, 720. [CrossRef]

23. Hetzel, U.; Szirovicza, L.; Smura, T.; Prähauser, B.; Vapalahti, O.; Kipar, A.; Hepojoki, J. Identification of a Novel Deltavirus in Boa Constrictors. mBio 2019, 10, e00014-19. [CrossRef]

24. Chang, W.S.; Pettersson, J.H.O.; Le Lay, C.; Shi, M.; Lo, N.; Wille, M.; Eden, J.S.; Holmes, E.C. Novel hepatitis D-like agents in vertebrates and invertebrates. Virus Evol. 2019, 5, vez021. [CrossRef] [PubMed]

25. Iwamoto, M.; Shibata, Y.; Kawasaki, J.; Kojima, S.; Li, Y.-T.; Iwami, S.; Muramatsu, M.; Wu, H.-L.; Wada, K.; Tomonaga, K.; et al. Identification of novel avian and mammalian deltaviruses provides new insights into deltavirus evolution. Virus Evol. 2021, 7, veab003. [CrossRef]

26. Bergner, L.M.; Orton, R.J.; Broos, A.; Tello, C.; Becker, D.J.; Carrera, J.E.; Patel, A.H.; Biek, R.; Streicker, D.G. Diversification of mammalian deltaviruses by host shifting. Proc. Natl. Acad. Sci. USA 2021, 118, e2019907118. [CrossRef]

27. Paraskevopoulou, S.; Pirzer, F.; Goldmann, N.; Schmid, J.; Corman, V.M.; Gottula, L.T.; Schroeder, S.; Rasche, A.; Muth, D.; Drexler, J.F.; et al. Mammalian deltavirus without hepadnavirus coinfection in the neotropical rodent Proechimys semispinosus. Proc. Natl. Acad. Sci. USA 2020, 117, 17977-17983. [CrossRef]

28. Gudima, S.; Wu, S.-Y.; Chiang, C.-M.; Moraleda, G.; Taylor, J. Origin of Hepatitis Delta Virus mRNA. J. Virol. 2000, 74, 7204-7210. [CrossRef] [PubMed]

29. De la Peña, M.; Ceprián, R.; Casey, J.L.; Cervera, A. Hepatitis delta virus-like circular RNAs from diverse metazoans encode conserved hammerhead ribozymes. Virus Evol. 2021, 7, veab016. [CrossRef]

30. Barrera, A.; Guerra, B.; Lee, H.; Lanford, R.E. Analysis of Host Range Phenotypes of Primate Hepadnaviruses by In Vitro Infections of Hepatitis D Virus Pseudotypes. J. Virol. 2004, 78, 5233-5243. [CrossRef]

31. Drexler, J.F.; Geipel, A.; König, A.; Corman, V.M.; van Riel, D.; Leijten, L.M.; Bremer, C.M.; Rasche, A.; Cottontail, V.M.; Maganga, G.D.; et al. Bats carry pathogenic hepadnaviruses antigenically related to hepatitis B virus and capable of infecting human hepatocytes. Proc. Natl. Acad. Sci. USA 2013, 110, 16151-16156. [CrossRef] [PubMed]

32. Gudima, S.; He, Y.; Chai, N.; Bruss, V.; Urban, S.; Mason, W.; Taylor, J. Primary Human Hepatocytes Are Susceptible to Infection by Hepatitis Delta Virus Assembled with Envelope Proteins of Woodchuck Hepatitis Virus. J. Virol. 2008, 82, 7276-7283. [CrossRef]

33. Szirovicza, L.; Hetzel, U.; Kipar, A.; Martinez-Sobrido, L.; Vapalahti, O.; Hepojoki, J. Snake Deltavirus Utilizes Envelope Proteins of Different Viruses to Generate Infectious Particles. mBio 2020, 11, e03250-19. [CrossRef] [PubMed]

34. Chen, H.-Y.; Shen, D.-T.; Ji, D.-Z.; Han, P.-C.; Zhang, W.-M.; Ma, J.-F.; Chen, W.-S.; Goyal, H.; Pan, S.; Xu, H.-G. Prevalence and burden of hepatitis D virus infection in the global population: A systematic review and meta-analysis. Gut 2019, 68, 512-521. [CrossRef]

35. Dény, P. Hepatitis Delta Virus Genetic Variability: From Genotypes I, II, III to Eight Major Clades? Curr. Top. Microbiol. Immunol. 2006, 307, 151-171. [CrossRef]

36. Huang, C.-R.; Lo, S.J. Evolution and Diversity of the Human Hepatitis D Virus Genome. Adv. Bioinform. 2010, $2010,323654$. [CrossRef] [PubMed]

37. Löytynoja, A.; Goldman, N. From the Cover: An algorithm for progressive multiple alignment of sequences with insertions. Proc. Natl. Acad. Sci. USA 2005, 102, 10557-10562. [CrossRef] [PubMed]

38. Löytynoja, A.; Goldman, N. Phylogeny-Aware Gap Placement Prevents Errors in Sequence Alignment and Evolutionary Analysis. Science 2008, 320, 1632-1635. [CrossRef]

39. Lefort, V.; Longueville, J.-E.; Gascuel, O. SMS: Smart Model Selection in PhyML. Mol. Biol. Evol. 2017, 34, 2422-2424. [CrossRef]

40. Letunic, I.; Bork, P. Interactive Tree of Life (iTOL): An online tool for phylogenetic tree display and annotation. Bioinformatics 2007, 23, 127-128. [CrossRef]

41. Griffin, B.L.; Chasovskikh, S.; Dritschilo, A.; Casey, J.L. Hepatitis Delta Antigen Requires a Flexible Quasi-Double-Stranded RNA Structure to Bind and Condense Hepatitis Delta Virus RNA in a Ribonucleoprotein Complex. J. Virol. 2014, 88, 7402-7411. [CrossRef]

42. Chang, M.F.; Chen, C.H.; Lin, S.L.; Chen, C.J.; Chang, S.C. Functional Domains of Delta Antigens and Viral-RNA Required for RNA Packaging of Hepatitis-Delta-Virus. J. Virol. 1995, 69, 2508-2514. [CrossRef]

43. Gudima, S.; Chang, J.; Moraleda, G.; Azvolinsky, A.; Taylor, J. Parameters of Human Hepatitis Delta Virus Genome Replication: The Quantity, Quality, and Intracellular Distribution of Viral Proteins and RNA. J. Virol. 2002, 76, 3709-3719. [CrossRef]

44. Hsieh, S.Y.; Taylor, J. Regulation of Polyadenylation of Hepatitis Delta-Virus Antigenomic RNA. J. Virol. 1991, 65, 6438-6446. [CrossRef]

45. Chen, P.J.; Kalpana, G.; Goldberg, J.; Mason, W.; Werner, B.; Gerin, J.; Taylor, J. Structure and Replication of the Genome of the Hepatitis Delta-Virus. Proc. Natl. Acad. Sci. USA 1986, 83, 8774-8778. [CrossRef]

46. Flores, R.; Grubb, D.; Elleuch, A.; Nohales, M.A.; Delgado, S.; Gago, S. Rolling-circle replication of viroids, viroid-like satellite RNAs and hepatitis delta virus: Variations on a theme. RNA Biol. 2011, 8, 200-206. [CrossRef] 
47. Macnaughton, T.B.; Lai, M.M.C. Genomic but Not Antigenomic Hepatitis Delta Virus RNA Is Preferentially Exported from the Nucleus Immediately after Synthesis and Processing. J. Virol. 2002, 76, 3928-3935. [CrossRef] [PubMed]

48. Lazinski, D.W.; Taylor, J.M. Expression of Hepatitis-Delta Virus-RNA Deletions-Cis and Trans Requirements for Self-Cleavage, Ligation, and RNA Packaging. J. Virol. 1994, 68, 2879-2888. [CrossRef] [PubMed]

49. Macnaughton, T.; Gowans, E.; Albert, A.; Burrell, C. Hepatitis delta virus RNA, protein synthesis and associated cytotoxicity in a stably transfected cell line. Virology 1990, 177, 692-698. [CrossRef]

50. Wang, Y.-H.; Chang, S.C.; Huang, C.; Li, Y.-P.; Lee, C.-H.; Chang, M.-F. Novel Nuclear Export Signal-Interacting Protein, NESI, Critical for the Assembly of Hepatitis Delta Virus. J. Virol. 2005, 79, 8113-8120. [CrossRef]

51. Glenn, J.S.; Watson, J.A.; Havel, C.M.; White, J.M. Identification of a prenylation site in delta virus large antigen. Science 1992, 256, 1331-1333. [CrossRef]

52. Komla-Soukha, I.; Sureau, C. A tryptophan-rich motif in the carboxyl-terminus of the small envelope protein of hepatitis B virus is central to the assembly of hepatitis delta virus particles. J. Virol. 2006, 80, 4648-4655. [CrossRef]

53. O'Malley, B.; Lazinski, D.W. Roles of Carboxyl-Terminal and Farnesylated Residues in the Functions of the Large Hepatitis Delta Antigen. J. Virol. 2005, 79, 1142-1153. [CrossRef]

54. Zuccola, H.J.; Rozzelle, J.E.; Lemon, S.M.; Erickson, B.W.; Hogle, J.M. Structural basis of the oligomerization of hepatitis delta antigen. Structure 1998, 6, 821-830. [CrossRef]

55. Yan, H.; Peng, B.; He, W.; Zhong, G.; Qi, Y.; Ren, B.; Gao, Z.; Jing, Z.; Song, M.; Xu, G.; et al. Molecular Determinants of Hepatitis B and D Virus Entry Restriction in Mouse Sodium Taurocholate Cotransporting Polypeptide. J. Virol. 2013, 87, 7977-7991. [CrossRef]

56. Yang, X.; Cai, W.; Sun, X.; Bi, Y.; Zeng, C.; Zhao, X.; Zhou, Q.; Xu, T.; Xie, Q.; Sun, P.; et al. Defined host factors support HBV infection in non-hepatic 293T cells. J. Cell. Mol. Med. 2020, 24, 2507-2518. [CrossRef]

57. Weller, M.L.; Gardener, M.R.; Bogus, Z.C.; Smith, M.A.; Astorri, E.; Michael, D.G.; Michael, D.A.; Zheng, C.; Burbelo, P.D.; Lai, Z.; et al. Hepatitis Delta Virus Detected in Salivary Glands of Sjogren's Syndrome Patients and Induces a Sjogren's Syndrome Phenotype In Vivo. Scand. J. Immunol. 2015, 81, 427.

58. Seeger, C.; Mason, W.S. Molecular biology of hepatitis B virus infection. Virology 2015, 479, 672-686. [CrossRef]

59. Freitas, N.; Cunha, C.; Menne, S.; Gudima, S.O. Envelope Proteins Derived from Naturally Integrated Hepatitis B Virus DNA Support Assembly and Release of Infectious Hepatitis Delta Virus Particles. J. Virol. 2014, 88, 5742-5754. [CrossRef]

60. Bichko, V.; Netter, H.J.; Taylor, J. Introduction of Hepatitis-Delta Virus into Animal-Cell Lines via Cationic Liposomes. J. Virol. 1994, 68, 5247-5252. [CrossRef]

61. Magnius, L.; Mason, W.S.; Taylor, J.; Kann, M.; Glebe, D.; Dény, P.; Sureau, C.; Norder, H.; ICTV Report Consortium. ICTV Virus Taxonomy Profile: Hepadnaviridae. J. Gen. Virol. 2020, 101, 571-572. [CrossRef]

62. Hahn, C.M.; Iwanowicz, L.R.; Cornman, R.S.; Conway, C.M.; Winton, J.R.; Blazer, V.S. Characterization of a Novel Hepadnavirus in the White Sucker (Catostomus commersonii) from the Great Lakes Region of the United States. J. Virol. 2015, 89, 11801-11811. [CrossRef] [PubMed]

63. Dill, J.A.; Camus, A.C.; Leary, J.H.; Di Giallonardo, F.; Holmes, E.C.; Ng, T.F.F. Distinct Viral Lineages from Fish and Amphibians Reveal the Complex Evolutionary History of Hepadnaviruses. J. Virol. 2016, 90, 7920-7933. [CrossRef] [PubMed]

64. Lauber, C.; Seitz, S.; Mattei, S.; Suh, A.; Beck, J.; Herstein, J.; Börold, J.; Salzburger, W.; Kaderali, L.; Briggs, J.A.; et al. Deciphering the Origin and Evolution of Hepatitis B Viruses by Means of a Family of Non-enveloped Fish Viruses. Cell Host Microbe 2017, 22, 387-399.e6. [CrossRef]

65. Freitas, N.; Salisse, J.; Cunha, C.; Toshkov, I.; Menne, S.; Gudima, S.O. Hepatitis delta virus infects the cells of hepadnavirusinduced hepatocellular carcinoma in woodchucks. Hepatology 2012, 56, 76-85. [CrossRef]

66. Flores, R.; Ruiz-Ruiz, S.; Serra, P. Viroids and Hepatitis Delta Virus. Semin. Liver Dis. 2012, 32, 201-210. [CrossRef]

67. Flores, R.; Owens, R.A.; Taylor, J. Pathogenesis by subviral agents: Viroids and hepatitis delta virus. Curr. Opin. Virol. 2016, 17, 87-94. [CrossRef]

68. Van Bogaert, N.; De Jonghe, K.; Van Damme, E.; Maes, M.; Smagghe, G. Quantitation and localization of pospiviroids in aphids. J. Virol. Methods 2015, 211, 51-54. [CrossRef]

69. Cappy, P.; Lucas, Q.; Kankarafou, N.; Sureau, C.; Laperche, S. No Evidence of Hepatitis C Virus (HCV)-Assisted Hepatitis D Virus Propagation in a Large Cohort of HCV-Positive Blood Donors. J. Infect. Dis. 2021, 223, 1376-1380. [CrossRef]

70. Chemin, I.; Pujol, F.H.; Scholtès, C.; Loureiro, C.L.; Amirache, F.; Levrero, M.; Zoulim, F.; Pérez-Vargas, J.; Cosset, F. Preliminary Evidence for Hepatitis Delta Virus Exposure in Patients Who Are Apparently Not Infected with Hepatitis B Virus. Hepatology 2021, 73, 861-864. [CrossRef]

71. Pfluger, L.S.; Schulze Zur Wiesch, J.; Polywka, S.; Lutgehetmann, M. Hepatitis delta virus propagation enabled by hepatitis C virus-Scientifically intriguing, but is it relevant to clinical practice? J. Viral Hepat. 2021, 28, 213-216. [CrossRef] 